\title{
Neural correlates of cognitive impairment in schizophrenia
}

Jordi Ortiz-Gil, Edith Pomarol-Clotet, Raymond Salvador, Erick J. Canales-Rodríguez, Salvador Sarró, Jesús J. Gomar, Amalia Guerrero, Bibiana Sans-Sansa, Antoni Capdevila, Carme Junqué and Peter J. McKenna

\section{Background}

Cognitive impairment is an established feature of schizophrenia. However, little is known about its relationship to the structural and functional brain abnormalities that characterise the disorder.

\section{Aims \\ To identify structural and/or functional brain abnormalities associated with schizophrenic cognitive impairment.}

\section{Method}

We carried out structural magnetic resonance imaging (MRI) and voxel-based morphometry in 26 participants who were cognitively impaired and 23 who were cognitively preserved, all with schizophrenia, plus 39 matched controls. Nineteen of those who were cognitively impaired and 18 of those who were cognitively preserved plus 34 controls also underwent functional MRI during performance of a working memory task.

\section{Results}

No differences were found between the participants who were cognitively intact and those who were cognitively impaired in lateral ventricular volume or whole brain volume. voxel-based morphometry also failed to reveal clusters of significant difference in grey and white matter volume between these two groups. However, during performance of the n-back task, the participants who were cognitively impaired showed hypoactivation compared with those who were cognitively intact in the dorsolateral prefrontal cortex among other brain regions.

\section{Conclusions}

Cognitive impairment in schizophrenia is not a function of the structural brain abnormality that accompanies the disorder but has correlates in altered brain function.

\section{Declaration of interest} None.
One of the most important changes in the concept of schizophrenia in recent years has been the recognition that cognitive impairment is part of the disorder. Although not a defining characteristic - some individuals are neurocognitively normal or near-normal ${ }^{1}$ - deficits similar in magnitude to those seen in central nervous system disease are common, ${ }^{2}$ and in a small number of cases may attain a severity comparable with dementia. ${ }^{3}$ Impairment is present in most or all areas of cognitive function but appears to be particularly marked in executive function and long-term memory. ${ }^{4}$ There are unanswered questions about the course of schizophrenic cognitive impairment, but the available evidence suggests that affected individuals show an IQ disadvantage compared with the rest of the population before they become ill; that a further decline in cognitive function takes place around illness onset; but that the level then remains stable, except in chronically hospitalised individuals in whom there may be a further decline in old age. ${ }^{5}$

Although cognitive impairment implies brain damage or dysfunction, little is known about the relationship between schizophrenic cognitive impairment and the structural and functional brain abnormalities that also characterise the disorder. Early computed tomography (CT) studies did not point consistently to an association with lateral ventricular enlargement. ${ }^{6}$ Reviewing magnetic resonance imaging (MRI) studies, Antonova et al found some evidence that whole brain, lateral ventricular and frontal and temporal lobe volume reductions were associated with general intellectual impairment and/or specific neuropsychological deficits, although there were conflicting findings in all cases. The findings were further complicated by gender differences in the associations found, and also by the existence of correlations between some volume measures and IQ in controls but not in participants with schizophrenia.
Techniques such as voxel-based morphometry (VBM), which map clusters of significant difference between groups of participants throughout the brain without the necessity of preselecting regions of interest, might have more power to detect small and/or localised volume differences related to cognitive impairment. Such studies have suggested that grey matter volume reductions are more extensive in individuals with chronic schizophrenia than in those with a first-episode, ${ }^{8,9}$ possibly in keeping with the finding that the former group typically show greater degrees of cognitive impairment than the latter. ${ }^{10,11}$ However, to date these techniques have not been used to examine the relationship between brain volume and cognitive impairment directly.

Investigation of the brain functional correlates of cognitive impairment in schizophrenia has been limited. In the first study to carry out functional imaging during performance of an executive task in schizophrenia, Weinberger et al ${ }^{12}$ found that the degree of hypofrontality correlated with the impairment the participants showed on the Wisconsin Card Sorting Test. However, such an association was not found in two later studies that used executive ${ }^{13}$ and memory ${ }^{14}$ tasks. Two meta-analyses of hypofrontality in schizophrenia have also examined the influence of task performance on prefrontal activation, ${ }^{15,16}$ and both found only trend-level correlations.

According to recent findings, schizophrenia is characterised not only by hypofrontality but also hyperfrontality, increased task-related activation in areas of the prefrontal cortex, which has been documented during performance of working memory ${ }^{17}$ and other executive tasks. ${ }^{18}$ Weinberger and colleagues ${ }^{19,20}$ have explicitly linked this latter finding to cognitive function, arguing that people with schizophrenia have to 'work harder to keep up' with task demands and so engage greater and/or more widespread 
cortical metabolic activity than those without schizophrenia when they try to do so. Nevertheless, a number of studies have compared participants with schizophrenia who are low- and high-performing on working memory tasks and their findings suggest that the relationship between hyperfrontality and cognitive impairment is quite complicated. ${ }^{21-23}$

To date, two studies have adopted a strategy of examining predefined groups of individuals with cognitive impairment. de Vries et $a l^{24}$ found that eight participants with schizophrenia and cognitive impairment amounting to dementia had no more ventricular enlargement or sulcal widening than that seen in schizophrenia as a whole. In contrast, most of the participants showed resting perfusion deficits on single photon emission computed tomography. Wexler et $a l^{25}$ found that 54 cognitively impaired people with schizophrenia showed similar degrees of lateral ventricular enlargement and grey matter volume reduction to 21 neuropsychologically near-normal individuals with the disorder. However, the cognitively impaired group had significantly smaller white matter volumes in two out of eight regions examined. This study did not investigate whether there were functional imaging differences between the two groups.

\section{Method}

\section{Participants}

Two groups of people with schizophrenia participated, one $(n=26)$ with and one $(n=23)$ without substantial degrees of cognitive impairment (the cognitively impaired group and cognitively preserved group respectively). Both these groups were recruited from long-stay wards $(n=14)$, acute and subacute units $(n=26)$ and out-patients/day hospital $(n=9)$. They all met DSM-IV $^{26}$ criteria for schizophrenia based on interview by two psychiatrists. Individuals were excluded if they were younger than 18 or older than 65 , had a history of brain trauma or neurological disease, or had shown alcohol/substance misuse within the 12 months prior to participation. Individuals were also excluded if they had a history of learning disability; this was based on attendance at a special school, or on an interview with relatives, for example if the estimated premorbid IQ measure was found to be low. All participants were taking antipsychotic medication (atypical $n=28$, typical $n=7$, both kinds $n=14$ ), and all were in a relatively stable clinical condition at the time of testing. The groups were selected to be matched for age, gender and premorbid IQ, as estimated using the Word Accentuation Test (TAP). ${ }^{27}$ This is conceptually similar to the National Adult Reading Test $(\mathrm{NART})^{28}$ and requires pronunciation of low-frequency Spanish words whose accents have been removed.

Presence of cognitive impairment was defined on the basis of performance on two well-standardised tests of memory and executive function, the Rivermead Behavioural Memory Test $(\mathrm{RBMT})^{29}$ and the Behavioural Assessment of the Dysexecutive Syndrome (BADS).$^{30}$ The RBMT consists of 12 subtests examining verbal recall, recognition, orientation, remembering a route and three measures of prospective memory, the ability to remember to do things. The BADS contains six subtests covering cognitive estimation, rule shifting, planning, problem-solving and decision-making under multiple task demands. The cognitively preserved group scored above the fifth percentile for normal adults on both tests (screening score of $\geqslant 8$ on the RBMT and profile score of $\geqslant 12$ on the BADS). The cognitively impaired group were required to score below the first percentile on either the RBMT (screening score of $<7$ ) or the BADS (profile score of $<8$ ).
The control group consisted of 39 healthy individuals recruited from the community. They met the same exclusion criteria and were selected to be matched to both the groups with schizophrenia in terms of age, gender and premorbid IQ. Controls were recruited from non-medical staff working in the hospital, their relatives and acquaintances, plus independent sources in the community. They were questioned and excluded if they reported a history of mental illness and/or treatment with psychotropic medication.

All participants were right-handed. They gave written informed consent and the study was approved by the local research ethics committee.

\section{Procedure}

All participants underwent structural and functional MRI (fMRI) scanning using the same 1.5 Tesla GE Signa scanner (General Electric Medical Systems, Milwaukee, USA).

\section{Structural imaging}

High-resolution structural $T_{1}$ MRI data were acquired with the following acquisition parameters: matrix size $512 \times 512$; 180 contiguous axial slices; voxel resolution $0.47 \times 0.47 \times 1 \mathrm{~mm}^{3}$; echo time $(\mathrm{TE})=3.93 \mathrm{~ms}$, repetition time $(\mathrm{TR})=2000 \mathrm{~ms}$ and inversion time $(\mathrm{TI})=710 \mathrm{~ms}$; flip angle $15^{\circ}$.

Calculation of the total volume of brain tissues (normalised for participant's head size) was performed with SIENAX, part of FSL (FMRIB Software Library, Oxford; www.fmrib.ox.ac.uk/ $\mathrm{fsl} /$ ). ${ }^{31,32}$ This tool additionally generates separate measures of grey and white matter volume. We compared lateral ventricle volume (also normalised for participant's head size) between groups using FreeSurfer (http://surfer.nmr.mgh.harvard. edu/fswiki), for which interrater reliability with manual segmentation has been shown. ${ }^{33}$

Structural data were further analysed with FSL-VBM, an optimised voxel-based morphometry style analysis ${ }^{34,35}$ carried out with FSL tools, which yields a measure of differences in local grey matter volume. First, structural images were brain-extracted. Next, tissue-type segmentation was carried out. The resulting grey matter partial volume images were then aligned to Montreal Neurologic Institute (MNI)152 standard space, followed by nonlinear registration. The resulting images were averaged to create a study-specific template, to which the native grey matter images were then non-linearly re-registered. The registered partial volume images were then modulated by dividing by the Jacobian of the warp field. The modulated segmentated images were then smoothed with an isotropic Gaussian kernel with a sigma of $4 \mathrm{~mm}$ (for technical details see www.fmrib.ox.ac.uk/fsl/fslvbm/). Group comparisons were carried out with permutation-based non-parametric tests. These were made with the randomise function implemented in FSL, using the recently developed threshold-free cluster-enhancement method with 10000 iterations, for proper statistical inference of spatially distributed patterns (corrected for multiple comparisons).

We also carried out a VBM analysis of white matter volume. Since the VBM analysis in FSL has only been validated for grey matter, we used VBM5 (http://dbm.neuro.uni-jena.de/vbm/ vbm5-for-spm5/), performed with SPM5 tools for this analysis. The following standard pre-processing steps were carried out: tissue-type segmentation; normalisation (warping) to standard space of the obtained white matter images; and modulation. The resulting images were then smoothed with an isotropic Gaussian kernel with a sigma of $4 \mathrm{~mm}$. Statistical analyses were carried out using the general linear model (GLM) with correction 
for multiple comparisons using the theory of Gaussian random fields.

\section{fMRI}

The paradigm used has been described by Pomarol-Clotet et al. ${ }^{36}$ Scanning was carried out while participants performed a sequential-letter version of the n-back task. ${ }^{37}$ Two levels of memory load (1-back and 2-back) were presented in a blocked design manner. Each block consisted of 24 letters that were shown every $2 \mathrm{~s}$ ( $1 \mathrm{~s}$ on, $1 \mathrm{~s}$ off) and all blocks contained 5 repetitions (1-back and 2-back depending on the block) located randomly within block. Participants had to indicate repetitions by pressing a button. Four 1-back and four 2-back blocks were presented in an interleaved way, and between these a baseline stimulus (an asterisk flashing with the same frequency as the letters) was presented for $16 \mathrm{~s}$. In order to identify which task had to be performed, characters were shown in green in 1-back blocks and in red in the 2-back blocks. All participants first went through a training session outside the scanner.

Performance was measured using the signal detection theory index of sensitivity $\left(\mathrm{d}^{\prime}\right) .^{38}$ Any participants who had negative $\mathrm{d}^{\prime}$ values in either or both of the 1-back and 2-back versions of the task, which suggests that they were not performing it, were excluded from the study.

In each individual scanning session 266 volumes were acquired. A gradient echo-planar imaging (EPI) sequence depicting the blood oxygen level-dependent (BOLD) contrast was used. Each volume contained 16 axial planes acquired with the following parameters: $\mathrm{TR}=2000 \mathrm{~ms}, \mathrm{TE}=20 \mathrm{~ms}$, flip angle $70^{\circ}$, section thickness $7 \mathrm{~mm}$, section skip $0.7 \mathrm{~mm}$, in-plane resolution $3 \times 3 \mathrm{~mm}^{2}$. The first ten volumes were discarded to avoid $T_{1}$ saturation effects.

Functional MRI analyses were performed with the FEAT module included in FSL software. ${ }^{32}$ At a first level, images were corrected for movement and coregistered to a common stereotaxic space (MNI template), and spatially filtered with a Gaussian filter (smoothing of full width at half maximum (FWHM) $5.0 \mathrm{~mm}$ ). To minimise unwanted movement-related effects, individuals with an estimated maximum absolute movement over $3.0 \mathrm{~mm}$, or an average absolute movement higher than $0.3 \mathrm{~mm}$ were discarded from the study. Finally, group comparisons were performed using the same FEAT module, by means of mixed-effects GLM models. A z-threshold of 2.3 (the default in FSL) was used to generate the initial set of clusters. To properly account for the spatially distributed patterns, FEAT uses the Gaussian random field theory when performing statistical tests.

\section{Data analysis}

The main focus in the structural and functional brain analyses was on two specific comparisons. First, we contrasted the cognitively preserved group with the control group. This was in order to determine changes in brain structure and function attributable to schizophrenia, without the complicating factor of cognitive impairment. Second, in order to assess the possible contribution of cognitive impairment itself, we contrasted the cognitively preserved and cognitively impaired groups. All statistical tests in the VBM and fMRI analyses were performed with a statistical threshold of $P<0.05$, corrected for multiple comparisons.

\section{Results}

\section{Sample characteristics}

There were no differences between the three groups in age, gender and TAP-estimated premorbid IQ (Table 1). The two groups with schizophrenia did not differ in overall severity of illness as measured by the Clinical Global Impression (CGI); ${ }^{40}$ however, the cognitively impaired group had significantly higher total symptom scores on the Positive and Negative Syndrome Scale (PANSS). ${ }^{41}$ They also had a significantly longer duration of illness than the cognitively preserved group and showed trend level higher mean dosages of antipsychotic drugs.

As expected, the two groups with schizophrenia differed significantly in their performance on the BADS and RBMT. The distributions of their scores are shown in Fig. 1. The cognitively impaired group also had lower scores on current IQ than the

Table 1 Demographic, neurocognitive and psychopathological characteristics of the participants with schizophrenia and controls

\begin{tabular}{|c|c|c|c|c|c|c|c|c|c|c|c|}
\hline & \multirow{3}{*}{$\begin{array}{l}\text { Control group } \\
\qquad(n=39)\end{array}$} & \multicolumn{2}{|c|}{$\begin{array}{l}\text { Participants with schizophrenia } \\
\qquad(n=49)\end{array}$} & \multicolumn{8}{|c|}{ Group statistics } \\
\hline & & \multirow{2}{*}{$\begin{array}{l}\text { Cognitively } \\
\text { preserved } \\
\text { group }(n=23)\end{array}$} & \multirow{2}{*}{$\begin{array}{l}\text { Cognitively } \\
\text { impaired } \\
\text { group }(n=26)\end{array}$} & \multicolumn{2}{|c|}{$\mathrm{I}<\mathrm{C}$} & \multicolumn{2}{|c|}{$\mathrm{I}<\mathrm{P}$} & \multirow[b]{2}{*}{$F$} & \multirow[b]{2}{*}{$\chi^{2}$} & $U$ & \multirow[b]{2}{*}{$P$} \\
\hline & & & & $t$ & $P$ & $t$ & $P$ & & & $t$ & \\
\hline Age, years: mean (s.d.) & $40.10(11.58)$ & $40.10(10.22)$ & $42.38(8.23)$ & & & & & 0.45 & & & 0.64 \\
\hline Gender, male/female: $n$ & $30 / 9$ & $17 / 6$ & $20 / 6$ & & & & & & 0.85 & & 0.96 \\
\hline TAP correct words, mean (s.d.) & $23.00(5.29)$ & $23.68(4.34)$ & $21.00(5.65)$ & & & & & 1.83 & & & 0.17 \\
\hline \multicolumn{12}{|l|}{ IQ (WAIS-III), mean (s.d.) } \\
\hline Full-scale IQ & $103.49(13.13)$ & $100.43(13.04)$ & $92.73(13.43)$ & 3.21 & 0.002 & 2.03 & 0.05 & 5.26 & & & 0.01 \\
\hline Verbal IQ & $104.90(16.73)$ & $104.00(17.65)$ & 96.85 (15.93) & & & & & 1.97 & & & 0.15 \\
\hline Performance IQ & 100.08 (17.59) & $94.00(14.61)$ & $84.54(16.56)$ & 3.57 & 0.001 & 2.11 & 0.04 & 6.87 & & & 0.002 \\
\hline BADS score, mean (s.d.) & & $16.04(2.40)$ & $10.69(4.33)$ & & & & & & & 5.43 & $<0.001$ \\
\hline RBMT screening score, mean (s.d.) & & $9.48(1.44)$ & $5.17(1.63)$ & & & & & & & 9.58 & $<0.001$ \\
\hline Years of illness, mean (s.d.) & & $18.28(10.02)$ & $23.76(8.29)$ & & & & & & & -2.09 & 0.04 \\
\hline PANSS total score, mean (s.d.) & & $66.57(17.11)$ & 76.15 (15.03) & & & & & & & -2.09 & 0.04 \\
\hline CGI score, mean (s.d.) & & $4.13(1.36)$ & $4.58(0.90)$ & & & & & & & 232.00 & 0.16 \\
\hline $\begin{array}{l}\text { Antipsychotic dosage } \\
\text { (chlorpromazine equivalent, mg), } \\
\text { mean (s.d.) }\end{array}$ & & $663.41(550.94)$ & $985.34(608.59)$ & & & & & & & -1.93 & 0.06 \\
\hline
\end{tabular}


(a)

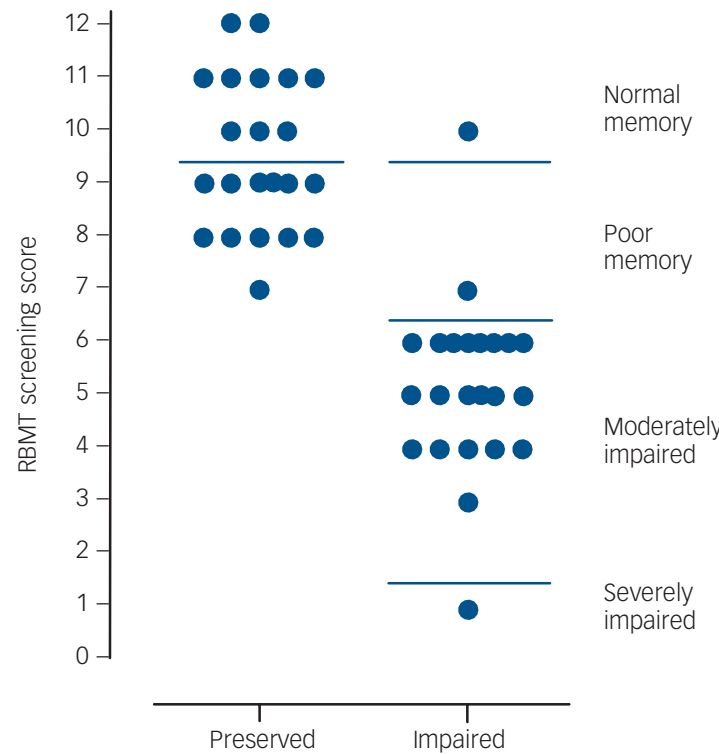

(b)

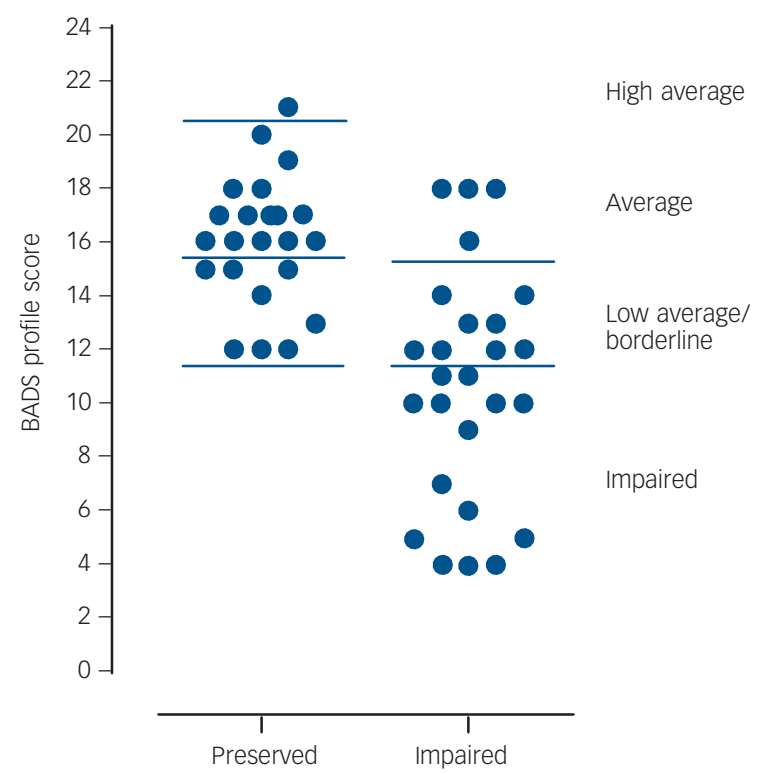

Fig 1 Scatter plots of the cognitively preserved and cognitively impaired groups' scores on the (a) Rivermead Behavioural Memory Test (RBMT) and (b) the Behavioural Assessment of the Dysexecutive Syndrome (BADS)

cognitively preserved group, but this only reached significance for performance IQ.

\section{Brain and lateral ventricular volume measures}

All participants were included in the analysis except in the comparison of lateral ventricles, where one control had to be excluded for technical reasons. Comparing all participants with schizophrenia with the controls, they showed reduced whole brain volume $\left(1526.75 \mathrm{~cm}^{3}\right.$ (s.d.=47.69) v. $1485.91 \mathrm{~cm}^{3}($ s.d. $=53.36)$, $t=3.74, P<0.001$, effect size $(\mathrm{ES})=0.80)$, reduced grey matter volume $\left(819.46 \mathrm{~cm}^{3}\right.$ (s.d. $\left.=35.39\right)$ v. $785.75 \mathrm{~cm}^{3} \quad$ (s.d. $\left.=39.09\right)$, $t=4.19, P<0.001, \mathrm{ES}=0.89)$ and lateral ventricular enlargement $\left(12.58 \mathrm{~cm}^{3}\right.$ (s.d.=7.24) v. $16.74 \mathrm{~cm}^{3}$ (s.d.=10.47), $t=-2.20$, $P=0.03$, ES $=-0.45)$. However, there was no difference in white matter volume between participants with schizophrenia and controls $\left(707.29 \mathrm{~cm}^{3}\right.$ (s.d. $\left.=25.62\right)$ v. $700.17 \mathrm{~cm}^{3}$ (s.d. $\left.=24.71\right)$, $t=1.32, P=0.19, \mathrm{ES}=0.28)$. As shown in Table 2, when the controls were compared with the cognitively preserved group the differences in whole brain and grey matter volume differences remained evident (whole brain: $t=2.62, P=0.01$, ES $=0.68$; grey matter: $t=2.83, P=0.006$, $\mathrm{ES}=0.73$ ), although that for lateral ventricular volume no longer reached significance $(t=-1.25$, $P=0.22$, ES $=-0.35)$. However, the differences between the cognitively preserved and cognitively impaired groups were small and non-significant on all these measures (whole brain: $t=0.36$, $P=0.72$, ES $=0.10$; grey matter: $t=0.62, P=0.53$, ES $=0.18$; lateral ventricular volume: $t=-0.92, P=0.36, \mathrm{ES}=-0.14$ ).

\section{VBM}

The same participants took part in this analysis, i.e. all those in the cognitively preserved group $(n=23)$ and cognitively impaired group $(n=26)$ and the 39 controls.

\section{Controls $v$. cognitively preserved group}

The cognitively preserved group showed significantly smaller grey matter volume than the controls in one cluster. This was situated anteriorly and medially, extending from the orbital and medial prefrontal cortex to the anterior cingulate gyrus (2190 voxels, $P=0.04$; peak in Brodmann Area (BA) 10 , MNI $(-12,44,-8)$, $z$-score $=4.70$ ). This is shown in Fig. 2 (the appearance of separate clusters is artefactual, due to the $3 \mathrm{D}$ rendering). There were no regions where the cognitively preserved group showed significantly greater volume than the controls.

No areas of significant white matter volume difference were found between the controls and the cognitively preserved group.

\section{Cognitively preserved group $v$. cognitively impaired group}

There were no areas of significant grey or white matter volume difference between these two groups.

\section{fMRI}

Some participants could not tolerate the fMRI procedure and in others the images were not usable because of excessive movement. Therefore, 19 participants who were cognitively impaired, 18 who were cognitively preserved and 34 controls took part in this analysis. As shown in Table 3, the groups remained matched for age, gender and TAP score. Significant differences between the two groups with schizophrenia remained evident on the BADS and the RBMT. These two groups did not differ in CGI or PANSS score, or in antipsychotic dosage. There were no significant differences between the participants with schizophrenia who took part in this part of the study and those who did not in terms of age (41.07 v. 42.05), gender (29/8v. 8/4) or TAP score (22.03 v. 22.83).

\section{Behavioural performance}

The cognitively preserved group were significantly impaired compared with the controls on the 1-back version of the task (mean d' 3.77 (s.d. $=0.91$ ) v. 4.40 (s.d. $=0.65$ ), $t=2.90, P=0.01$ ) and in the 2-back version (mean $\mathrm{d}^{\prime} 2.67$ (s.d. $=0.87$ ) v. 3.27 (s.d. $=0.96), t=2.22, P=0.03)$. The cognitively impaired group were marginally significantly impaired compared with the cognitively preserved group on the 1-back task (mean d' 3.07 $($ s.d. $=1.16) \quad$ v. $3.77 \quad($ s.d. $=0.91), \quad t=2.03, \quad P=0.05) \quad$ and significantly impaired on the 2-back task (mean d' 1.89 (s.d. $=0.68)$ v. 2.67 (s.d. $=0.87), t=3.06, P=0.004$ ). 


\begin{tabular}{|c|c|c|c|c|c|c|c|c|c|c|c|}
\hline & \multirow[b]{3}{*}{ Controls $(n=39)$} & \multirow{3}{*}{$\begin{array}{l}\text { Cognitively } \\
\text { preserved group } \\
\qquad(n=23)\end{array}$} & \multirow{3}{*}{$\begin{array}{l}\text { Cognitively } \\
\text { impaired group } \\
(n=26)\end{array}$} & \multicolumn{8}{|c|}{ ANOVA } \\
\hline & & & & \multicolumn{2}{|c|}{$\mathrm{P}<\mathrm{C}$} & \multicolumn{2}{|c|}{$\mathrm{I}<\mathrm{C}$} & \multicolumn{2}{|c|}{$I>C$} & \multirow[b]{2}{*}{$F$} & \multirow[b]{2}{*}{$P$} \\
\hline & & & & $t$ & $P$ & $t$ & $P$ & $t$ & $P$ & & \\
\hline Whole brain & $1526.75(47.69)$ & $1488.82(65.92)$ & 1483.35 (40.36) & 2.62 & 0.01 & 3.82 & $<0.001$ & & & 6.98 & 0.002 \\
\hline Grey matter & 819.46 (35.39) & 789.55 (47.52) & 782.38 (30.36) & 2.83 & 0.006 & 4.37 & $<0.001$ & & & 8.94 & $<0.001$ \\
\hline White matter & $707.29(25.62)$ & 699.27 (29.79) & 700.96 (19.74) & & & & & & & 0.89 & 0.41 \\
\hline Lateral ventricles $^{a}$ & $12.58(7.24)$ & 15.95 (12.49) & $17.44(8.49)$ & & & & & -2.59 & 0.01 & 2.95 & 0.06 \\
\hline
\end{tabular}

Controls $v$. cognitively preserved group

No areas of significant difference in activation were seen in the 1-back $v$. baseline contrast or in the 2-back $v$. 1-back contrast. In the 2-back $v$. baseline contrast the controls activated more than the cognitively preserved group in the right cerebellum (1606 voxels, $P=8.27 \times 10^{-5}$, MNI $(12,-58,-24), z$-score 4.48$)$.

Additionally, in the 2-back $v$. baseline contrast, the cognitively preserved group showed two clusters where they failed to de-activate significantly relative to the control group. The larger of these included parts of the medial and inferior orbital prefrontal cortex, extending to the anterior cingulate cortex (3878 voxels, $P=1.72 \times 10^{-9}$, peak activation in BA11, MNI $(0,26,-14), z$-score 4.52). The smaller cluster was located in the right insula and in the right superior temporal gyrus $(629$ voxels, $P=0.04$, peak activation in BA48, MNI $(42,-8,-6), z$-score 4.13$)$.

This failure of de-activation was more evident in the 2-back $v$. 1-back contrast. Here, a large cluster was seen that included the medial and inferior orbital prefrontal cortex, the left basal ganglia and anterior regions of the left temporal cortex (5748 voxels, $P=8.66 \times 10^{-13}$, peak activation in BA38, MNI $(-40,18$, $-34), z$-score 4.49). Another cluster affected parts of the right basal ganglia and anterior temporal cortex (2235 voxels, $P=2.56 \times 10^{-6}$; peak activation in BA35, MNI $(26,2,-34), z$-score 4.56) (Fig. 3).

\section{Cognitively preserved group $v$. cognitively impaired group}

There were no differences between the groups in the 1-back $v$. baseline contrast. The 2-back $v$. baseline contrast revealed significantly reduced activation in the cognitively impaired group in an area that included the right dorsolateral prefrontal cortex, the inferior lateral frontal lobe and the right insula (1749 voxels, $P=2.94 \times 10^{-5}$, peak activation in right frontal inferior pars triangularis, MNI $(38,28,26), z$-score 3.93). This area of reduced
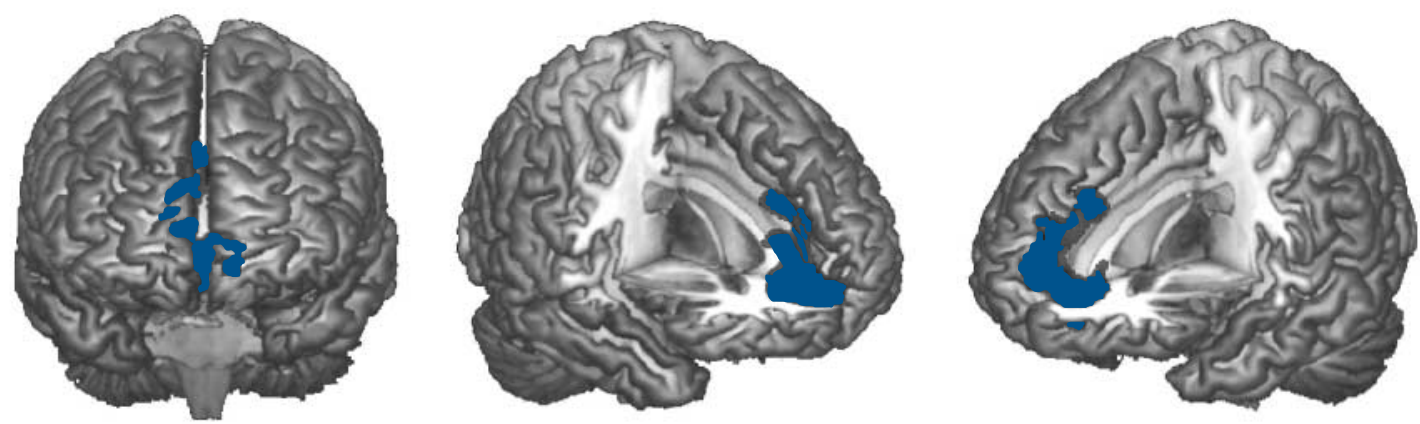

Fig. 2 Brain regions showing significant grey matter volume reduction in the cognitively preserved group with schizophrenia compared with healthy controls.
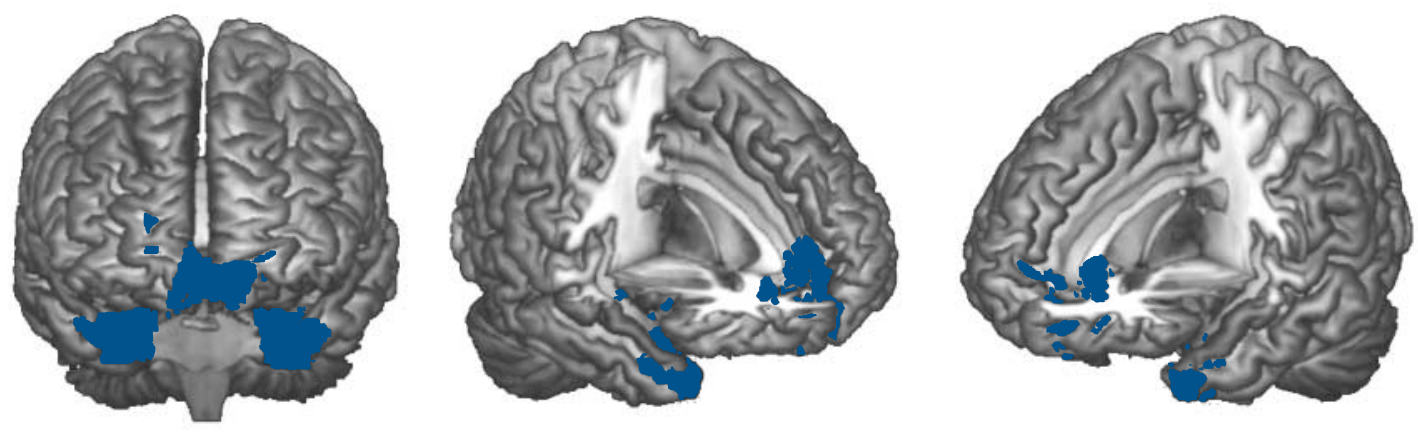

Fig. 3 Brain regions where the cognitively preserved group with schizophrenia showed significant failure to de-activate compared with the controls in the 2-back v. 1-back contrast. 


\begin{tabular}{|c|c|c|c|c|c|c|c|c|c|c|c|c|}
\hline & \multirow{3}{*}{$\begin{array}{l}\text { Control group } \\
\qquad(n=34)\end{array}$} & \multicolumn{2}{|c|}{$\begin{array}{l}\text { Participants with schizophrenia } \\
\qquad(n=37)\end{array}$} & \multicolumn{9}{|c|}{ Group statistics } \\
\hline & & \multirow{2}{*}{$\begin{array}{l}\text { Cognitively } \\
\text { preserved } \\
\text { group }(n=18)\end{array}$} & \multirow{2}{*}{$\begin{array}{l}\text { Cognitively } \\
\text { impaired } \\
\text { group }(n=19)\end{array}$} & \multicolumn{2}{|c|}{$\mathrm{I}<\mathrm{C}$} & \multicolumn{2}{|c|}{$\mathrm{I}<\mathrm{P}$} & \multirow[b]{2}{*}{$F$} & \multirow[b]{2}{*}{$\chi^{2}$} & \multirow[b]{2}{*}{$t$} & \multirow[b]{2}{*}{$U$} & \multirow[b]{2}{*}{$P$} \\
\hline & & & & $t$ & $P$ & $t$ & $P$ & & & & & \\
\hline Age, years: mean (s.d.) & $40.90(11.80)$ & $40.49(10.58)$ & $41.62(7.94)$ & & & & & 0.06 & & & & 0.95 \\
\hline Gender, male/female: $n$ & $26 / 8$ & $14 / 4$ & $15 / 4$ & & & & & & 0.04 & & & 0.98 \\
\hline TAP correct words, mean (s.d.) & $23.00(5.42)$ & $23.41(4.02)$ & $20.79(5.08)$ & & & & & 1.55 & & & & 0.25 \\
\hline \multicolumn{13}{|l|}{ IQ (WAIS-III), mean (s.d.) } \\
\hline Full-scale IQ & $104.24(12.47)$ & $100.44(13.99)$ & $94.11(9.37)$ & 3.08 & 0.003 & & & 4.24 & & & & 0.02 \\
\hline Verbal IQ & $105.44(16.06)$ & $103.06(19.07)$ & $96.58(10.86)$ & & & & & 1.95 & & & & 0.15 \\
\hline Performance IQ & 100.85 (18.19) & $94.67(15.68)$ & $86.74(17.08)$ & 2.77 & 0.01 & & & 4.09 & & & & 0.02 \\
\hline BADS score, mean (s.d.) & & $16.06(2.69)$ & $11.58(4.26)$ & & & & & & & 3.80 & & 0.001 \\
\hline RBMT screening score, mean (s.d.) & & $9.72(1.36)$ & $5.56(1.46)$ & & & & & & & 8.84 & & 0.001 \\
\hline Years of illness, mean (s.d.) & & $18.44(10.86)$ & $22.71(7.71)$ & & & & & & & -1.39 & & 0.18 \\
\hline PANSS total score, mean (s.d.) & & $67.89(18.33)$ & $76.79(17.04)$ & & & & & & & -1.53 & & 0.14 \\
\hline CGI score, mean (s.d.) & & $4.28(1.41)$ & $4.58(1.02)$ & & & & & & & & 146.50 & 0.44 \\
\hline $\begin{array}{l}\text { Antipsychotic dosage } \\
\text { (chlorpromazine equivalent, mg), } \\
\text { mean (s.d.) }\end{array}$ & & $688.22(603.25)$ & $913.50(507.21)$ & & & & & & & -1.23 & & 0.23 \\
\hline
\end{tabular}

activation was more pronounced in the 2-back $v$. 1-back contrast: on the right, one cluster included the dorsolateral prefrontal cortex extending to the precentral gyrus posteriorly and the superior middle frontal cortex anteriorly (2494 voxels, $P=1.19 \times 10^{-7}$, peak activation in BA42, MNI $(12,24,46), z$-score 3.88). A similar cluster on the left included the dorsolateral prefrontal cortex and extended to the basal ganglia, the insula and the precentral gyrus (1786 voxels, $P=96 \times 10^{-6}$; peak activation in BA6, MNI $(-30,6,24), z$-score 4.27). Two more clusters were located in regions of the right parietal and occipital lobes (1962 voxels, $P=2.09 \times 10^{-6}$, peak activation in BA40, MNI $(38,-46,50), z$-score 4.25$)$ and in roughly similar regions on the left ( 1785 voxels, $P=6.02 \times 10^{-6}$, peak activation in BA7, MNI $(-32,-64,48), z$-score 3.91). Two further small clusters were found in both thalami (608 voxels, $P=0.02$, peak activation in the right thalamus, MNI $(6,-8,19), z$-score 2.9$)$ and in the left inferior and middle occipital gyri (603 voxels, $P=0.03$, peak activation in BA19, MNI $(-52,-76,-2), z$-score 4.04). The findings are shown in Fig. 4.

There were no areas where the cognitively impaired group activated more than the cognitively preserved group.

\section{Discussion}

\section{Structural imaging findings}

As a group, the participants with schizophrenia in this study showed typical structural imaging findings associated with the disorder, namely reduced brain volume, reduced grey matter volume and lateral ventricular enlargement. However, the cognitively preserved and cognitively impaired groups did not differ from each other on these measures. When VBM was used to examine grey and white matter volume further, a cluster of grey matter volume reduction was seen in the cognitively preserved group in the medial and orbital prefrontal cortex, overlapping with areas identified in recent meta-analyses. ${ }^{9,42}$ Once again, no clusters of significant grey or white matter volume difference emerged between the cognitively preserved and cognitively impaired groups.

Although counterintuitive, these findings are consistent with the rest of the structural imaging literature, which has documented only weak and conflicting evidence of an association between cognitive impairment and lateral ventricular size, whole brain volume and regional cortical volumes in schizophrenia. ${ }^{6,7}$
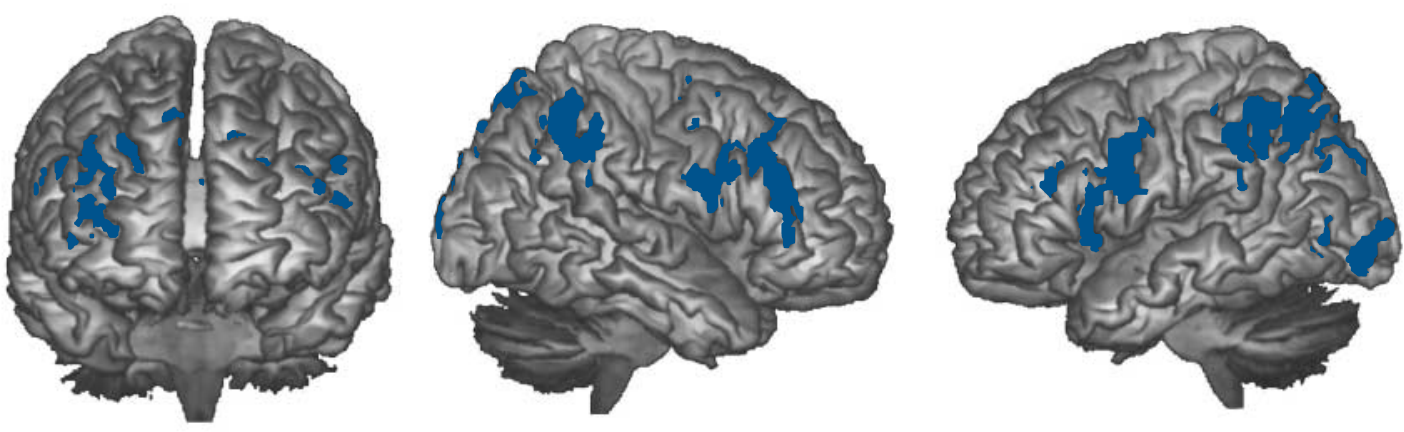

Fig. 4 Brain regions where the cognitively preserved group activated significantly more than the cognitively impaired group in the 2-back v. 1-back contrast. 
The recent study of Wexler et $a l^{25}$ the only other study besides ours to explicitly compare groups of cognitively preserved and impaired individuals with schizophrenia, also failed to find significant differences in lateral ventricular volume and grey matter volume between them. Wexler et $a l^{25}$ did find that cognitively impaired individuals showed significantly smaller white matter volume in two out of eight regions examined (sensorimotor and parietal-occipital cortex). However, these differences may not have been robust since there was no control for multiple comparisons.

Our structural imaging findings are also in keeping with a well-established neuropathological finding in schizophrenia. This is that, although severe cognitive impairment is prevalent among elderly people who are institutionalised - more than $70 \%$ have Mini-Mental State Examination (MMSE) scores in the demented range ${ }^{43}$ - post-mortem studies have revealed no more Alzheimer-type or other brain pathology in such individuals than in age-matched controls. ${ }^{3}$

Nevertheless, our study does not completely exclude the possibility of small structural differences related to cognitive function. This is because in the conventional MRI analysis there were differences in whole brain volume and grey matter volume between the cognitively impaired and cognitively preserved groups of $0.4 \%$ and $0.9 \%$ respectively. Although these differences were small and non-significant, the reductions of brain volume in schizophrenia as a whole are also small, being of the order of $2 \%$ (whole brain) and 4\% (grey matter) according to the metaanalysis of Wright et al. ${ }^{44}$ It could therefore be argued that our study was simply underpowered to detect differences between the two groups with schizophrenia. However, it should be noted that two groups of 769 participants would be required to make the differences we found in whole brain volume between cognitively impaired and cognitive preserved groups significant, and 239 for each group would be needed to do so for the differences in grey matter volume.

A final objection to our finding of no relationship between cognitive impairment and brain volume reduction is conceptual. If, as is widely accepted, ${ }^{45}$ structural brain abnormality in schizophrenia is neurodevelopmental in origin, then it might not be expected to show the same relationship with cognitive impairment as brain changes that are the result of brain injury or degenerative disease. When the evidence that additional brain volume reductions also take place after illness onset ${ }^{46}$ is also taken into account, plus the fact that cognitive impairment itself follows a complex pre-, peri- and postmorbid course, ${ }^{5}$ there is scope for a further argument, that the relationship between brain structure and cognitive impairment in schizophrenia cannot be adequately assessed in a simple cross-sectional study such as ours.

\section{Functional imaging findings}

In contrast to the brain structural findings, we found clear evidence of differences between the cognitively impaired and cognitively preserved groups on functional imaging. Specifically, in the 2-back $v$. baseline contrast the cognitively impaired group showed reduced activation compared with the cognitively preserved group in the right dorsolateral prefrontal cortex and other frontal areas, changes which became bilateral and extended more widely in the 2-back $v$. 1-back contrast. In fact, most of the task-related hypoactivation we found appeared to be attributable to cognitive impairment - in the comparison between the cognitively preserved group and the controls the cognitively preserved group showed reduced activation only in the cerebellum.

This result deviates somewhat from the rest of the literature which, as noted in the introduction, has not found evidence of a robust correlation between hypofrontality and task performance. ${ }^{15,16}$ One possible reason for our stronger findings here is that, rather than using correlational methods, we prospectively compared groups that differed in cognitive function but which were matched for other factors that might affect task performance, especially premorbid intellectual function. The fact that the two groups were also well-separated in terms of memory and/or executive performance (i.e. one was above the fifth percentile and the other was below the first percentile) would also have tended to increase functional imaging differences between them related to this factor.

It does not seem likely that the differences we found between the cognitively impaired and cognitively preserved groups were the result of the former simply not performing the task, since we excluded a priori any participants who showed negative $\mathrm{d}^{\prime}$ scores, an indicator of failure to perform the task. At the same time, the difference in level of n-back performance between the two groups with schizophrenia has the potential to complicate the interpretation of any functional imaging differences found between them. This possibility could not be investigated in our study because the groups were preselected on the basis that they differed in cognitive function and the n-back task is itself a cognitive task. Therefore, entering n-back performance as a covariate in the analysis would have violated the principle that the covariate should not be affected by the group factor.

In fact, this issue is part of a wider debate about what drives task-related hypofrontality in schizophrenia: are both poor task performance and reduced brain activation manifestations of an underlying intrinsic cortical dysfunction? Or does the reduced activation merely index the fact that cognitively impaired individuals perform the task more poorly and so activate their frontal lobes to a correspondingly lesser extent (see Fletcher et $\left.a l^{14}\right)$ ? This debate has now to some extent been superseded by the finding that schizophrenia is characterised not only by hypofrontality, but also by hyperfrontality during task performance. ${ }^{17,18}$ Nevertheless, cognitive impairment continues to play a central role in explanations of this latter functional imaging abnormality. Thus, according to Weinberger et al, ${ }^{19,20}$ people with schizophrenia have reduced efficiency of prefrontal cortical processing. This causes them to show more activation than healthy individuals - i.e. hyperfrontality - at low task demands, as they 'work harder to keep up'. As task demands increase, they then reach their limit of performance sooner than healthy participants, and thereafter show a fall-off of activation, or hypofrontality. We did not find any evidence of hyperfrontality in our study, suggesting that this abnormality may not be related to cognitive function in the way predicted by Weinberger and colleagues, ${ }^{19,21}$ a conclusion also reached by Karlsgodt et al. ${ }^{23}$ However, it should be noted that we did not fully examine this question, since the theory predicts that hyperfrontality should be seen at low task difficulty in the comparison between controls and individuals who are cognitively impaired, and we did not compare these two groups directly.

In addition to reduced activation related to cognitive function, we also found failure of de-activation. This affected the medial frontal cortex among other areas and, since it was only seen in the comparison between the controls and the cognitively preserved group, it was unrelated to the presence of cognitive impairment. Failure of task-related de-activation in the medial frontal cortex in schizophrenia has now been documented several times, ${ }^{36,47,48}$ where it has been interpreted as evidence of dysfunction in the default mode network - one of the two prominent midline nodes of which is located in the medial frontal cortex. The default mode network is currently a focus of considerable research interest in schizophrenia, with studies 
finding evidence of both changes in task-related de-activation and abnormal connectivity at rest (for a review see Broyd et $a l^{49}$ ). Among other things, it has been suggested that failure of de-activation in the network might account for the cognitive impairment associated with the schizophrenia. ${ }^{36,47}$ Our findings suggest that this is not the case.

Also interesting in this respect was the overlap between the structural and functional abnormalities that was evident in our study: in the VBM comparison between the controls and the cognitively preserved group, volume reductions were clustered in a medial frontal cortex region where failure of de-activation was also seen. We have previously examined this overlap in more detail, ${ }^{50}$ and two other studies have had comparable findings. Camchong et $a l^{51}$ found functional connectivity abnormality in the anterior node of the default mode network, plus white matter changes in subjacent regions on diffusion tensor imaging, and Salgado-Pineda et $a l^{48}$ found failure of both de-activation and volume reductions in regions extending along the length of the cingulate gyrus.

\section{Conclusions and limitations}

This study provides evidence that structural brain abnormality in schizophrenia is a function of having the disorder, not the cognitive impairment that goes with it. In contrast, a substantial part of the functional imaging abnormality associated with schizophrenia appears to reflect cognitive impairment. Limitations of the study include the relatively small sizes of the groups with and without cognitive impairment. Also, since the cognitively preserved group was defined in terms of memory and executive function above fifth percentile cut-offs, it was not completely free of cognitive impairment; some fell into the poor normal memory range on the RBMT and the low average/ borderline categories in the BADS. As discussed above, the inferences that can be drawn from positive findings in an fMRI comparison between cognitively preserved and cognitively impaired individuals are inevitably limited by the differences in performance between them on the task used. In general terms, more detailed knowledge about the trajectories of structural and functional brain change in schizophrenia might be needed before firm conclusions can be drawn about their relationship with cognitive impairment in the disorder.

Jordi Ortiz-Gil, MSC, Benito Menni Complex Assistencial en Salut Mental, Barcelona,
CIBERSAM, and Medicine PhD program, University of Barcelona, Barcelona; Edith
Pomarol-Clotet, MD, PhD, Germanes Hospitalaries, FIDMAG, Barcelona and
CIBERSAM; Raymond Salvador, PhD, Benito Menni Complex Assistencial en Salut
Mental, Barcelona, CIBERSAM, and Fundació Sant Joan de Déu, Barcelona; Erick J.
Canales-Rodríguez, BSC, Benito Menni Complex Assistencial en Salut Mental,
Barcelona and CIBERSAM; Salvador Sarró, MD, Germanes Hospitalaries, FIDMAG,
Barcelona and CIBERSAM; Jesús J. Gomar, MSC, Benito Menni Complex Assistencial
en Salut Mental, Barcelona and CIBERSAM; Amalia Guerrero, MD, Bibiana
Sans-Sansa, MSC, Benito Menni Complex Assistencial en Salut Mental, Barcelona;
Antoni Capdevila, MD, Hospital de Sant Pau and Fundació Sant Joan de Déu,
Barcelona; Carme Junqué, PhD, Medicine PhD program, University of Barcelona,
Barcelona and Department of Psychiatry and Clinical Psychobiology, University of
Barcelona, Barcelona, Spain; the August Pi i Sunyer Biomedical Research Institute
(IDIBAPS), Barcelona; Peter J. McKenna, MPCPsych, Benito Menni Complex
Assistencial en Salut Mental, Barcelona and CIBERSAM, Spain

Correspondence: P. J. McKenna, Benito Menni Complex Assistencial en Salut Mental. Germanes Hospitalàries del Sagrat Cor de Jesús, C/ Doctor Antoni

Pujades 38-C, 08830 - Sant Boi de Llobregat, Barcelona, Spain. Email: mckennapeter1@googlemail.com

First received 10 Jun 2010, final revision 21 Feb 2011, accepted 21 Mar 2011

\section{Funding}

Supported by the Instituto de Salud Carlos III, Centro de Investigación en Red de Salud Mental, CIBERSAM and Marie Curie European Reintegration Grant (MERG-CT-2004511069) given to E.P.-C. Four grants from the Spanish Ministry of Health - Instituto de Salud
Carlos III: PI05/2693 provided to E.P.-C.; CP07/00048, PI05/1874 given to R.S.; F105/00322 given to J.G.; CM07/00016 given to B.S-S.; CA06/0129 given to J.O.-G.

\section{References}

1 Kremen WS, Seidman LJ, Faraone SV, Toomey R, Tsuang MT. The paradox of normal neuropsychological function in schizophrenia. J Abnorm Psychol 2000; 109: 743-52.

2 Heinrichs RW, Zakzanis KK. Neurocognitive deficit in schizophrenia: a quantitative review of the evidence. Neuropsychology 1998; 12: 426-45.

3 Harrison PJ. The neuropathology of schizophrenia. A critical review of the data and their interpretation. Brain 1999; 122: 593-624.

4 Reichenberg A, Harvey PD. Neuropsychological impairments in schizophrenia: integration of performance-based and brain imaging findings. Psychol Bull 2007; 133: 833-58.

5 Palmer BW, Dawes SE, Heaton RK. What do we know about neuropsychological aspects of schizophrenia? Neuropsychol Rev 2009; 19: $365-84$.

6 Lewis SW. Computerised tomography in schizophrenia 15 years on. Br J Psychiatry 1990; 157 (suppl 9): 16-24.

7 Antonova E, Sharma T, Morris R, Kumari V. The relationship between brain structure and neurocognition in schizophrenia: a selective review. Schizophr Res 2004; 70: 117-45.

8 Ellison-Wright I, Glahn DC, Laird AR, Thelen SM, Bullmore E. The anatomy of first-episode and chronic schizophrenia: an anatomical likelihood estimation meta-analysis. Am J Psychiatry 2008; 165: 1015-23.

9 Bora E, Fornito A, Radua J, Walterfang $\mathrm{M}$, Seal M, Wood SJ, et al. Neuroanatomical abnormalities in schizophrenia: a multimodal voxelwise meta-analysis and meta-regression analysis. Schizophr Res 2011; 127: 46-57.

10 Heaton RK, Baade LE, Johnson KL. Neuropsychological test results associated with psychiatric disorders in adults. Psychol Bull 1978; 85: 141-62.

11 Saykin AJ, Shtasel DL, Gur RE, Stafiniak P, Kester DB, Mozley LH, et al. Neuropsychological deficits in neuroleptic naive patients with first-episode schizophrenia. Arch Gen Psychiatry 1994; 51: 124-31.

12 Weinberger DR, Berman KF, Zec RF. Physiologic dysfunction of dorsolateral prefrontal cortex in schizophrenia. I. Regional cerebral blood flow evidence. Arch Gen Psychiatry 1986; 43: 114-24.

13 Frith $C D$, Friston KJ, Herold S, Silbersweig D, Fletcher P, Cahill C, et al. Regional brain activity in chronic schizophrenic patients during the performance of a verbal fluency task. Br J Psychiatry 1995; 167: 343-9.

14 Fletcher PC, McKenna PJ, Frith CD, Grasby PM, Friston KJ, Dolan RJ. Brain activations in schizophrenia during a graded memory task studied with functional neuroimaging. Arch Gen Psychiatry 1998; 55: 1001-8.

15 Hill K, Mann L, Laws KR, Stephenson CM, Nimmo-Smith I, McKenna PJ. Hypofrontality in schizophrenia: a meta-analysis of functional imaging studies. Acta Psychiatr Scand 2004; 110: 243-56.

16 Van Snellenberg JX, Torres IJ, Thornton AE. Functional neuroimaging of working memory in schizophrenia: task performance as a moderating variable. Neuropsychology 2006; 20: 497-510.

17 Glahn DC, Ragland JD, Abramoff A, Barrett J, Laird AR, Bearden CE, et al. Beyond hypofrontality: a quantitative meta-analysis of functional neuroimaging studies of working memory in schizophrenia. Hum Brain Mapp 2005; 25: 60-9.

18 Minzenberg MJ, Laird AR, Thelen S, Carter CS, Glahn DC. Meta-analysis of 41 functional neuroimaging studies of executive function in schizophrenia. Arch Gen Psychiatry 2009; 66: 811-22.

19 Tan HY, Callicott JH, Weinberger DR. Dysfunctional and compensatory prefrontal cortical systems, genes and the pathogenesis of schizophrenia. Cereb Cortex 2007; 17 (suppl 1): i171-81.

20 Weinberger DR, Egan MF, Bertolino A, Callicott JH, Mattay VS, Lipska BK, et al. Prefrontal neurons and the genetics of schizophrenia. Biol Psychiatry 2001; 50: $825-44$.

21 Callicott JH, Mattay VS, Verchinski BA, Marenco S, Egan MF, Weinberger DR. Complexity of prefrontal cortical dysfunction in schizophrenia: more than up or down. Am J Psychiatry 2003; 160: 2209-15.

22 Tan HY, Sust S, Buckholtz JW, Mattay VS, Meyer-Lindenberg A, Egan MF, et al. Dysfunctional prefrontal regional specialization and compensation in schizophrenia. Am J Psychiatry 2006; 163: 1969-77.

23 Karlsgodt KH, Sanz J, van Erp TG, Bearden CE, Nuechterlein KH, Cannon TD. Re-evaluating dorsolateral prefrontal cortex activation during working memory in schizophrenia. Schizophr Res 2009; 108: 143-50.

24 de Vries PJ, Honer WG, Kemp PM, McKenna PJ. Dementia as a complication of schizophrenia. J Neurol Neurosurg Psychiatr 2001; 70: 588-96. 
25 Wexler BE, Zhu H, Bell MD, Nicholls SS, Fulbright RK, Gore JC, et al. Neuropsychological near normality and brain structure abnormality in schizophrenia. Am J Psychiatry 2009; 166: 189-95.

26 American Psychiatric Association. Diagnostic and Statistical Manual of Mental Disorders (4th edn) (DSM-IV). APA, 1994.

27 Del Ser T, Gonzalez-Montalvo Jl, Martinez-Espinosa S, Delgado-Villapalos C Bermejo F. Estimation of premorbid intelligence in Spanish people with the Word Accentuation Test and its application to the diagnosis of dementia. Brain Cogn 1997; 33: 343-56.

28 Nelson HE, Willison JR. The Revised National Adult Reading Test. nferNelson, 1991

29 Wilson B, Cockburn J, Baddeley A, Hiorns R. The Rivermead Behavioural Memory Test. Thames Valley Test Company, 1985.

30 Wilson BA, Burgess PW, Emslie H, Evans JJ. Behavioural Assessment of the Dysexecutive Syndrome (BADS). Thames Valley Test Company, 1996.

31 Smith SM. Fast robust automated brain extraction. Hum Brain Mapp 2002; 17: $143-55$.

32 Smith SM, Jenkinson M, Woolrich MW, Beckmann CF, Behrens TE, JohansenBerg $\mathrm{H}$, et al. Advances in functional and structural MR image analysis and implementation as FSL. Neuroimage 2004; 23 (suppl 1): s208-19.

33 Fischl B, Dale AM. Measuring the thickness of the human cerebral cortex from magnetic resonance images. Proc Natl Acad Sci U S A 2000; 97 : 11050-5.

34 Ashburner J, Friston KJ. Voxel-based morphometry - the methods. Neuroimage 2000; 11: 805-21.

35 Good CD, Johnsrude IS, Ashburner J, Henson RN, Friston KJ, Frackowiak RS A voxel-based morphometric study of ageing in 465 normal adult human brains. Neuroimage 2001; 14: 21-36.

36 Pomarol-Clotet E, Salvador R, Sarro S, Gomar J, Vila F, Martínez A, et al. Failure to deactivate in the prefrontal cortex in schizophrenia: dysfunction of the default mode network? Psychol Med 2008; 38: 1185-93.

37 Gevins A, Cutillo B. Spatiotemporal dynamics of component processes in human working memory. Electroencephalogr Clin Neurophysiol 1993; 87 128-43.

38 Green DM, Swets JA. Signal Detection Theory And Psychophysics. Krieger, 1966.

39 Wechsler D. Wecshler Adult Intelligence Scale (3rd edn). The Psychological Corporation, 1997
40 GUy WE. ECDEU Assessment Manual for Psychopharmacology. US Department of Health, Education, and Welfare, 1976.

41 Kay SR, Fiszbein A, Opler LA. The positive and negative syndrome scale (PANSS) for schizophrenia. Schizophr Bull 1987; 13: 261-76.

42 Fornito A, Yucel M, Patti J, Wood SJ, Pantelis C. Mapping grey matter reductions in schizophrenia: an anatomical likelihood estimation analysis of voxel-based morphometry studies. Schizophr Res 2009; 108: 104-13.

43 Harvey PD, Lombardi J, Kincaid MM, Parrella M, White L, Powchik P, et al. Cognitive functioning in chronically hospitalized schizophrenic patients: age-related changes and age disorientation as a predictor of impairment. Schizophr Res 1995; 17: 15-24.

44 Wright IC, Rabe-Hesketh S, Woodruff PW, David AS, Murray RM, Bullmore ET. Meta-analysis of regional brain volumes in schizophrenia. Am J Psychiatry 2000; 157: 16-25.

45 Weinberger DR, Marenco S. Schizophrenia as a neurodevelopmental disorder. In Schizophrenia (2nd edn) (eds SR Hirsch, DR Weinberger): 326-48. Blackwell, 2003.

46 Hulshoff Pol HE, Kahn RS. What happens after the first episode? A review of progressive brain changes in chronically ill patients with schizophrenia. Schizophr Bull 2008; 34: 354-66.

47 Whitfield-Gabrieli S, Thermenos HW, Milanovic S, Milanovic S, Tsuang MT, Faraone SV, et al. Hyperactivity and hyperconnectivity of the default network in schizophrenia and in first-degree relatives of persons with schizophrenia. Proc Natl Acad Sci U S A 2009; 106: 1279-84.

48 Salgado-Pineda P, Fakra E, Delaveau P, McKenna PJ, Pomarol-Clotet E, Blin O. Correlated structural and functional brain abnormalities in the default mode network in schizophrenia patients. Schizophr Res 2011; 125: 101-9.

49 Broyd SJ, Demanuele C, Debener S, Helps SK, James CJ, Sonuga-Barke EJ. Default-mode brain dysfunction in mental disorders: a systematic review. Neurosci Biobehav Rev 2009; 33: 279-96.

50 Pomarol-Clotet E, Canales-Rodriguez EJ, Salvador R, Sarró S, Gomar JJ, Vila F, et al. Medial prefrontal cortex pathology in schizophrenia as revealed by convergent findings from multimodal imaging. Mol Psychiatry 2010; 15: 82330.

51 Camchong J, Macdonald 3rd AW, Bell C, Mueller BA, Lim KO. Altered functional and anatomical connectivity in schizophrenia. Schizophr Bull 2009; 37: $640-50$. 